\title{
THERMOELASTIC INTERACTIONS IN A ROTATING INFINITE ORTHOTROPIC ELASTIC BODY WITH A CYLINDRICAL HOLE AND VARIABLE THERMAL CONDUCTIVITY
}

\begin{abstract}
In the present article, we introduced a new model of the equations of generalized thermoelasticity for unbounded orthotropic body containing a cylindrical cavity. We applied this model in the context of generalized thermoelasticity with phase-lags under the effect of rotation. In this case, the thermal conductivity of the material is considered to be variable. In addition, the cylinder surface is traction free and subjected to a uniform unit step temperature. Using the Laplace transform technique, the distributions of the temperature, displacement, radial stress and hoop stress are determined. A detailed analysis of the effects of rotation, phase-lags and the variability thermal conductivity parameters on the studied fields is discussed. Numerical results for the studied fields are illustrated graphically in the presence and absence of rotation.
\end{abstract}

\section{Introduction}

In material science, building insulation, electronics, research, and associated fields, particularly where high operational temperatures are achieved, variability of thermal conductivity is very important. The effect of temperature on thermal conductivity is distinctive for metals and nonmetals. Thermal conductivity of metals is around relative to the absolute temperature (in Kelvin) times the electrical conductivity. It is to be noted that the electrical conductivity in pure metals dimin-

\footnotetext{
${ }^{1}$ Department of Mathematics, Faculty of Science, King Abdulaziz University, P.O. Box 80203, Jeddah 21589,Saudi Arabia.Emails:_mashat@kau.edu.sa, zenkour@kau.edu.sa

${ }^{2}$ Department of Mathematics, Faculty of Science, Kafrelsheikh University, Kafrelsheikh 33516, Egypt.Email:zenkour@sci.kfs.edu.eg

${ }^{3}$ Department of Mathematics, College of Science and Arts, Aljouf University, Al-Qurayat, Saudi Arabia; Department of Mathematics, Faculty of Science, Mansoura University, Mansoura 35516, Egypt.Email: ahabogal@gmail.com
} 
ishes with expanding temperature, the thermal conductivity remains approximately constant. In amalgams the change in electrical conductivity is normally smaller and in this way thermal conductivity increments with temperature, regularly relatively to temperature [1].

The generalized theories of thermoelasticity have created to defeat unbounded proliferation speed of thermal signals, which was predicted in classical dynamical coupled theory of thermoelasticity. Biot [2] built up the coupled model of thermoelasticity which tackles the first shortcoming of the uncoupled theory, however, it shares the second shortcoming of the uncoupled theory. Lord and Shulman [3] presented generalized theory thermoelasticity with one relaxation time by proposing another law of heat transfer to change traditional Fourier's law. This law includes heat flux vector and also its time derivative. Green and Lindsay [4] developed a theory that contains two constants times that act as relaxation times and modifies not the heat conduction equation only but also all the equations of the coupled theory. Zenkour [5] presented a comparison between various generalized thermoelasticity theories to discuss three-dimensional thermal shock plate problem. Zenkour [6-8] restricts his attention to the theory of Green and Naghdi of type III to deal with nano-machined beam resonators subjected to various boundary conditions and those resting on visco-Pasternak's foundations. Tzou [9] suggested a dual phase delay model of the heat conduction (DPL) to combine the effects of infinitesimal interactions in the fast-transient procedure of heat transportation mechanism in a macroscopic design. Two diverse phase-lags have been presented in the constitutive relation among the vector of heat flux and the gradient of the temperature [10].

Abouelregal [11] studied a piezo-thermo-electric half-space medium with dependent properties of temperature under a ramp-type changing heating using the generalized theory with fractional order. Zenkour and Abouelregal [12] introduced a generalized new model of the nonlocal generalized thermoelasticity for nanobeams due to a harmonically changing in heat taking into consideration the variability of thermal conductivity. Zenkour et al. [13] discussed the generalized theory of the nonlocal thermoelasticity presented by Lord and Shulman [3] to study the Euler-Bernoulli nanobeams vibrations assuming the temperature-dependent thermal conductivity. Ezzat and El-Bary [14] studied the fractional derivative effects in a perfect conducting infinite hollow cylinder and considering that the thermal conductivity is variable.

It has been shown up there that rotation causes thermoelastic generalized medium to be diffusive and having a physical property that has different values when measured in different directions. This treatise joined some examination on the phenomenon of the free surface in a rotating medium. It gives the idea that little consideration has been paid to examination of propagation of plane thermoelastic waves in a rotating medium.

Many investigations have been devoted to studies on the rotation effect of thermoelastic propagation of waves in an isotropic infinite cylinder materials. Abouelregal and Abo-Dahab $[15,16]$ introduced the effect of dual phase lag model on a non- 
homogeneous magneto-thermoelastic infinite solid having a spherical cavity and studied the diffusion effect and Thomson's phenomenon on magneto-thermoelastic solid cylinder. Abouelregal and Zenkour [17] concerned the effect of rotation on an isotropic homogeneous thermoelastic half-space due to a crack of Mode-I using the generalized theory of thermoelasticity. Singh and Kumar [18] explained the rotation effect on micropolar magneto-thermoelastic body. Xiong and Guo [19] investigated the effect of heat source moving with constant speed, and variable properties of a magneto-thermoelastic medium, under the fractional order theory of thermoelasticity. Kumar et al. [20] analyzed the interactions subjected to the effect of rotation and hall current in a magneto- thermoelastic micropolar half-space under the fractional order thermoelasticity. Sherief and Hamza [21] considered the variability of thermal conductivity effect on a magneto-thermoelastic infinitely hollow cylinder.

In the current article, a problem of an infiite homogeneous orthotropic thermally conducting body containing a cylindrical cavity affected by the angular velocity is discussed in the context of Tzou model. The thermal conductivity of the body is thought to be changing with the temperature [22-24]. The surface of the cylinder is exposed to thermal shock that depends on the time and surface of the body drop free. The outcomes for generalized and classical theories of thermoelasticity have been obtained from the resultant model as special cases. To explain and compare the theoretical results, the numerical solution is done by means of Laplace transform technique. The variability effects of thermal conductivity, rotation, phase lags on distributions of displacements, temperature and stresses are displayed graphically.

\section{The basic equations}

We consider an infinite orthotropic, homogeneous, isotropic conducting thermoelastic rotating body with density $\rho$ at initial constant temperature $T_{0}$. The field equations in generalized thermoelasticity with dual-phase-lags in the absence of the body force are $[25,26]$ :

equations of motion:

$$
C_{i j m n} \varepsilon_{m n, j}-\beta_{i j} \theta_{, j}+F_{i}=\rho \ddot{u}_{i}+\rho[\vec{\Omega} \times(\vec{\Omega} \times \vec{u})]_{i}+2 \rho(\vec{\Omega} \times \overrightarrow{\dot{u}})_{i} .
$$

Constitutive relations are

$$
\sigma_{i j}=C_{i j m n} \varepsilon_{m n}-\beta_{i j} \theta \delta_{i j}, \quad i, j=1,2,3,
$$

where

$$
\varepsilon_{i j}=\frac{1}{2}\left(u_{i, j}+u_{j, i}\right)
$$


The generalized heat conduction equation in the context of dual-phase-lag theory (DPL) suggested by Tzou is given by

$$
\left(1+\tau_{\theta} \frac{\partial}{\partial t}\right)\left(K_{i j} \theta_{, j}\right)_{, i}=\left(\delta+\tau_{q} \frac{\partial}{\partial t}\right)\left(\rho C_{E} \frac{\partial \theta}{\partial t}+\beta_{i j} T_{0} \frac{\partial u_{m, m}}{\partial t}-\rho Q\right) .
$$

In all the above governing equations (1)-(4), an over dot indicates the partial derivative as for the time variable, $\theta=T-T_{0}$ represents the thermodynamical temperature, $\delta_{i j}$ is Kronecker's delta, $\beta_{i j}$ are the thermoelastic components of the coupling, $C_{i j m n}$ are isothermal elastic constants, $\sigma_{i j}$ are the stress tensor components, $F_{i}$ are the body force components, $\vec{\Omega}=\Omega \vec{n}$ is the angular velocity, $\vec{n}$ is the direction of the axis of rotation, $\vec{\Omega} \times(\vec{\Omega} \times \vec{u})$ is the centripetal acceleration, $2(\vec{\Omega} \times \vec{u})$ is the Coriolis acceleration and $u_{i}$ are the displacement vector components. In addition, $\varepsilon_{i j}$ symbolizes the strain tensor, $\varepsilon_{k k}=e$ is cubical dilatation, $K_{i j}$ is the thermal conductivity tensor which is considered to be temperature-dependent, $C_{E}$ represents the specific heat of the body at constant strain, $Q$ is the heat source, $\tau_{q}$ and $\tau_{\theta}$ are the phase-lags of the heat flux and gradient of temperature, respectively, such that $0 \leqslant \tau_{\theta}<\tau_{q}$.

The coupled and generalized theories thermoelasticity can be obtained as limited cases depending on the values of $\delta, \tau_{q}$ and $\tau_{\theta}$. Putting $\tau_{\theta}=0, \delta=1$, and $\tau_{q}=\tau_{0}$ (the first relaxation time), gives the basic equations of governing Lord and Shulman's theory (LS). Also, when $\tau_{q}=\tau_{\theta}=0$, governing equation for a classical thermoelastic body (CTE) are obtained.

\section{The problem formulation}

In this section, we study an infinite thermoelastic orthotropic body with cylindrical cavity. The surface of cavity is exposed to a time dependent varying heat and traction free. We take the cylindrical coordinates $(r, \xi, z)$ with the $z$-axis considering all the functions depending on the radial space $r$ and the variable time $t$ according to the symmetry about $z$-axis.

For axially symmetric problem, the displacement vector has its components

$$
u_{r}=u(r, t), \quad u_{\xi}(r, t)=u_{z}(r, t)=0 .
$$

Consequently, the radial and hoop strains $\varepsilon_{r r}$ and $\varepsilon_{\xi \xi}$ are given by:

$$
\varepsilon_{r r}=\frac{\partial u}{\partial r}, \quad \varepsilon_{\xi \xi}=\frac{u}{r}
$$

The sum of normal strains gives the cubic dilatation

$$
e=\varepsilon_{r r}+\varepsilon_{\xi \xi}=\frac{\partial u}{\partial r}+\frac{u}{r} .
$$


The stress-displacement relations (2) can be obtained after using Eq. (6) as

$$
\left\{\begin{array}{c}
\left(\sigma_{r r}\right. \\
\sigma_{\xi \xi} \\
\sigma_{z z}
\end{array}\right\}=\left[\begin{array}{lll}
c_{11} & c_{12} & -\beta_{11} \\
c_{12} & c_{22} & -\beta_{22} \\
c_{13} & c_{23} & -\beta_{33}
\end{array}\right]\left\{\begin{array}{c}
\frac{\partial u}{\partial r} \\
\frac{u}{r} \\
\theta
\end{array}\right\} .
$$

Taking the rotation term about the $z$-axis as a body force only, Eq. (1) in the $r$ direction will be in the following form

$$
\frac{\partial \sigma_{r r}}{\partial r}+\frac{\sigma_{r r}-\sigma_{\xi \xi}}{r}=\rho \frac{\partial^{2} u}{\partial t^{2}}-\rho \Omega^{2} u
$$

where $\Omega$ is the uniform angular velocity. Introducing Eqs. (8) into equation of motion (9), we get

$$
c_{11}\left(\frac{\partial^{2} u}{\partial r^{2}}+\frac{1}{r} \frac{\partial u}{\partial r}\right)-c_{22} \frac{u}{r^{2}}=\rho \frac{\partial^{2} u}{\partial t^{2}}+\beta_{11} \frac{\partial \theta}{\partial r}+\left(\beta_{11}-\beta_{22}\right) \frac{\theta}{r}-\rho \Omega^{2} u .
$$

Also, the one-dimensional generalized heat equation (4) can be obtained as

$$
\left(1+\tau_{\theta} \frac{\partial}{\partial t}\right)\left(K_{r} \theta_{, r}\right)_{, r}=\left(\delta+\tau_{q} \frac{\partial}{\partial t}\right)\left[\rho C_{E} \frac{\partial \theta}{\partial t}+T_{0} \frac{\partial}{\partial t}\left(\beta_{11} \frac{\partial u}{\partial r}+\beta_{22} \frac{u}{r}\right)\right],
$$

where the thermal conductivity $K_{r}$ is temperature dependence, the above heat Eq. (11) is nonlinear of temperature and possibly of specific heat $C_{E}$.

\section{Temperature-dependent thermal conductivity}

Experimentally, the thermal properties of the material differ with temperature and this translates into a nonlinear heat equation and nonlinear thermoelastic problem, so that the temperature dependence of material properties must be contemplated in the thermal stress investigation of these components [27-30]. The solution of the nonlinear problems can be observed and obtained by supposing the material to be temperature dependent implying that the specific heat $C_{E}$ and the $r$ direction thermal conductivity $K_{r}$ depend on the distribution of temperature [31]. Additionally, the experimental information demonstrates that variations of Poisson's ratio and the thermal expansion coefficient, because of the high temperature, can be dismissed [31].

Assuming that the thermal conductivity $K_{r}$ is a linear function of temperature $\theta[32]:$

$$
K_{r}=K_{r}(\theta)=k_{0}\left(1+k_{1} \theta\right),
$$

where $k_{0}$ is the thermal conductivity at initial temperature $T_{0}$ and $k_{1}$ is a factor characterizing the variety of thermal conductivity. 
To solve the problem, we will take the following transformation [32]

$$
\psi=\frac{1}{k_{0}} \int_{0}^{\theta} K_{r}(\theta) \mathrm{d} \theta
$$

where the new function $\psi$ expressing the heat conduction. Substituting from Eq. (12) into Eq. (13) and integrating, we obtain [32]

$$
\psi=\theta\left(1+\frac{1}{2} k_{1} \theta\right)
$$

From Eqs. (13) and (14), we deduce the following two relations

$$
\begin{aligned}
& \nabla \psi=\frac{K_{r}(\theta)}{k_{0}} \nabla \theta, \\
& \frac{\partial \psi}{\partial t}=\frac{K_{r}(\theta)}{k_{0}} \frac{\partial \theta}{\partial t} .
\end{aligned}
$$

The generalized heat conduction equation (11) of thermally conducting orthotropic, homogeneous solids with variable thermal conductivity after using Eqs. (15) and (16) reduce to

$$
\left(1+\tau_{\theta} \frac{\partial}{\partial t}\right) \nabla^{2} \psi=\left(1+\tau_{q} \frac{\partial}{\partial t}\right)\left[\rho C_{E} \frac{\partial \psi}{\partial t}+\frac{T_{0}}{K_{0}} \frac{\partial}{\partial t}\left(\beta_{11} \frac{\partial u}{\partial r}+\beta_{22} \frac{u}{r}\right)\right],
$$

where $\rho C_{E}=K_{r} / k$ and

$$
\nabla^{2}=\frac{\partial^{2}}{\partial r^{2}}+\frac{1}{r} \frac{\partial}{\partial r}
$$

Substituting from Eqs. (15) and (16) into motion equation (10), we have

$$
\begin{aligned}
c_{11}\left(\frac{\partial^{2} u}{\partial r^{2}}+\frac{1}{r} \frac{\partial u}{\partial r}\right)-c_{22} \frac{u}{r^{2}}= & \rho \frac{\partial^{2} u}{\partial t^{2}}+\frac{\beta_{11}}{1+2 k_{1} \theta} \frac{\partial \psi}{\partial r}-\rho \Omega^{2} u \\
& +\frac{\beta_{11}-\beta_{22}}{k_{1} r}\left[\sqrt{1+2 k_{1} \psi}-1\right] .
\end{aligned}
$$

Assuming that $\theta=T-T_{0}$ is small and does not cause insignificant variations of elastic and thermal coefficients, these will be regarded as independent of $T$. In addition to the assumption $\left|\theta / T_{0}\right| \ll 1$, one can assume that second powers and products of the components of strain may be neglected in comparison with the strains $\varepsilon_{i j}$.

For linearity and by means of the binomial theorem for fractional powers and the assumption $\left|\theta / T_{0}\right| \ll 1$, the radial equation of motion (19) and constitutive relations (8) will be in the forms

$$
c_{11}\left(\frac{\partial^{2} u}{\partial r^{2}}+\frac{1}{r} \frac{\partial u}{\partial r}\right)-c_{22} \frac{u}{r^{2}}=\rho \frac{\partial^{2} u}{\partial t^{2}}+\beta_{11} \frac{\partial \psi}{\partial r}+\left(\beta_{11}-\beta_{22}\right) \frac{\psi}{r}-\rho \Omega^{2} u,
$$




$$
\left\{\begin{array}{l}
\sigma_{r r} \\
\sigma_{\xi \xi} \\
\sigma_{z z}
\end{array}\right\}=\left[\begin{array}{lll}
c_{11} & c_{12} & -\beta_{11} \\
c_{12} & c_{22} & -\beta_{22} \\
c_{13} & c_{23} & -\beta_{33}
\end{array}\right]\left\{\begin{array}{c}
\frac{\partial u}{\partial r} \\
\frac{u}{r} \\
\psi
\end{array}\right\} .
$$

Presenting the following dimensionless variables,

$$
\begin{gathered}
\left\{r^{\prime}, u^{\prime}, R^{\prime}\right\}=\frac{c_{0}}{k}\{r, u, R\}, \quad\left\{t^{\prime}, \tau_{q}^{\prime}, \tau_{\theta}^{\prime}\right\}=\frac{c_{0}^{2}}{k}\left\{t, \tau_{q}, \tau_{\theta}\right\}, \\
\psi^{\prime}=\frac{\psi}{T_{0}}, \quad \Omega^{\prime}=\frac{\Omega}{\eta c_{0}^{2}}, \quad \sigma_{i j}^{\prime}=\frac{\sigma_{i j}}{c_{11}}, \quad k_{1}^{\prime}=T_{0} k_{1}, \quad c_{0}^{2}=\frac{c_{11}}{\rho},
\end{gathered}
$$

the dimensionless basic equations are given by

$$
\begin{gathered}
\frac{\partial^{2} u}{\partial r^{2}}+\frac{1}{r} \frac{\partial u}{\partial r}-c_{2} \frac{u}{r^{2}}=\frac{\partial^{2} u}{\partial t^{2}}+\varepsilon_{1} \frac{\partial \psi}{\partial r}+\varepsilon_{3} \frac{\psi}{r}-\Omega^{2} u \\
\left(1+\tau_{\theta} \frac{\partial}{\partial t}\right) \nabla^{2} \psi=\left(1+\tau_{q} \frac{\partial}{\partial t}\right)\left[\frac{\partial \psi}{\partial t}+\frac{\partial}{\partial t}\left(\left(\varepsilon_{4} \frac{\partial u}{\partial r}+\varepsilon_{5} \frac{u}{r}\right)\right],\right. \\
\left\{\begin{array}{l}
\sigma_{r r} \\
\sigma_{\xi \xi} \\
\sigma_{z z}
\end{array}\right\}=\left[\begin{array}{lll}
1 & c_{1} & -\varepsilon_{1} \\
c_{1} & c_{2} & -\varepsilon_{2} \\
c_{3} & c_{4} & -\varepsilon_{6}
\end{array}\right]\left\{\begin{array}{c}
\frac{\partial u}{\partial r} \\
\frac{u}{r} \\
\psi
\end{array}\right\},
\end{gathered}
$$

where

$$
\begin{gathered}
\left\{\varepsilon_{1}, \varepsilon_{2}, \varepsilon_{3}, \varepsilon_{6}\right\}=\frac{T_{0}}{c_{11}}\left\{\beta_{11}, \beta_{22},\left(\beta_{11}-\beta_{22}\right), \beta_{33}\right\}, \\
\left\{\varepsilon_{4}, \varepsilon_{5}\right\}=\frac{1}{\rho C_{E}}\left\{\beta_{11}, \beta_{22}\right\}, \quad\left\{c_{1}, c_{2}, c_{3}, c_{4}\right\}=\frac{1}{c_{11}}\left\{c_{12}, c_{22}, c_{13}, c_{23}\right\} .
\end{gathered}
$$

\section{Application and boundary conditions}

We take the conditions that initially occurred in the problem as

$$
u=\left.\frac{\partial u}{\partial t}\right|_{t=0}=0, \quad \psi=\left.\frac{\partial \psi}{\partial t}\right|_{t=0}=0 .
$$

Also, one can consider that the medium is quiet and the cylindrical surface is subjected to the following boundary conditions:

- Thermal shock varying heat

$$
\theta(r, t)=\theta_{0} H(t), \quad r=R, \quad \theta_{0}=\text { const. }, \quad t>0 .
$$


From Eq. (16), then boundary condition (28) is given by

$$
\psi(R, t)=\theta_{0} H(t)+\frac{1}{2} k_{1}\left[\theta_{0} H(t)\right]^{2} .
$$

- The cylindrical surface is traction free, i.e.,

$$
\sigma_{r r}(r, t)=0, \quad r=R
$$

\section{Solution in Laplace's transform domain}

Laplace's transform is utilized to change governing equations from the time domain into Laplace and space field. Assume that the underlying conditions for dimensionless temperature and displacement are zero. Applying Laplace's transformation

$$
\bar{f}(r, s)=\int_{0}^{\infty} \mathrm{e}^{-s t} f(r, t) \mathrm{d} t,
$$

under the initial conditions (27) to Eqs. (23)-(25) and assuming that $\beta_{11}=\beta_{22}$ (i.e., $\varepsilon_{4}=\varepsilon_{5}=\varepsilon$ ) and $c_{11}=c_{22}$. That is

$$
\begin{gathered}
\frac{\mathrm{d}^{2} \bar{u}}{\mathrm{~d} r^{2}}+\frac{1}{r} \frac{\mathrm{d} \bar{u}}{\mathrm{~d} r}-\frac{\bar{u}}{r^{2}}-\left(s^{2}-\Omega^{2}\right) \bar{u}=\varepsilon_{1} \frac{\mathrm{d} \bar{\psi}}{\mathrm{d} r}, \\
\frac{\mathrm{d}^{2} \bar{\psi}}{\mathrm{d} r^{2}}+\frac{1}{r} \frac{\mathrm{d} \bar{\psi}}{\mathrm{d} r}=\frac{s\left(1+\tau_{q} s\right)}{1+\tau_{\theta} s}\left[\bar{\psi}+\varepsilon\left(\frac{\mathrm{d} \bar{u}}{\mathrm{~d} r}+\frac{\bar{u}}{r}\right)\right], \\
\left\{\begin{array}{l}
\bar{\sigma}_{r r} \\
\bar{\sigma}_{\xi \xi} \\
\bar{\sigma}_{z z}
\end{array}\right\}=\left[\begin{array}{lll}
1 & c_{1} & -\varepsilon_{1} \\
c_{1} & c_{2} & -\varepsilon_{2} \\
c_{3} & c_{4} & -\varepsilon_{6}
\end{array}\right]\left\{\begin{array}{l}
\frac{\mathrm{d} \bar{u}}{\mathrm{~d} r} \\
\bar{u} \\
\bar{r} \\
\bar{\psi}
\end{array}\right\} .
\end{gathered}
$$

Equations (32) and (33) can be reduces to

$$
\begin{gathered}
\left(D D_{1}-s^{2}+\Omega^{2}\right) \bar{u}=\varepsilon_{1} D \bar{\psi}, \\
\varepsilon q D_{1} \bar{u}=\left(D_{1} D-q\right) \bar{\psi},
\end{gathered}
$$

where

$$
D=\frac{\mathrm{d}}{\mathrm{d} r}, \quad D_{1}=\frac{\mathrm{d}}{\mathrm{d} r}+\frac{1}{r}, \quad q=\frac{s\left(1+\tau_{q} s\right)}{1+\tau_{\theta} s} .
$$

Presenting the potential function $\phi(r)$, defined by

$$
u=\frac{\mathrm{d} \phi}{\mathrm{d} r}
$$


into reduced equations (35) and (36), thus we have

$$
\begin{aligned}
& \left(D D_{1}-s^{2}+\Omega^{2}\right) \bar{\phi}=\varepsilon_{1} \bar{\psi}, \\
& \varepsilon q D_{1} D \bar{\phi}=\left(D_{1} D-q\right) \bar{\psi} .
\end{aligned}
$$

Combining Eqs. (39) and (40) one obtains the equation

$$
\left\{\nabla^{4}-\left[s^{2}-\Omega^{2}+q\left(1+\varepsilon_{1} \varepsilon\right)\right] \nabla^{2}+q\left(s^{2}-\Omega^{2}\right)\right\} \bar{\phi}=0 .
$$

If $m_{1}^{2}$ and $m_{2}^{2}$ are roots of the specific equation

$$
m^{4}-\left[s^{2}-\Omega^{2}+q\left(1+\varepsilon_{1} \varepsilon\right)\right] m^{2}+q\left(s^{2}-\Omega^{2}\right)=0
$$

we can factorize Eq. (41) as

$$
\left(\nabla^{2}-m_{1}^{2}\right)\left(\nabla^{2}-m_{2}^{2}\right) \bar{\phi}=0 .
$$

The roots $m_{1}^{2}$ and $m_{2}^{2}$ are obtained as

$$
m_{1}^{2}=\frac{1}{2}\left(2 A+\sqrt{A^{2}-4 B}\right), \quad m_{2}^{2}=\frac{1}{2}\left(2 A-\sqrt{A^{2}-4 B}\right),
$$

where

$$
A=s^{2}-\Omega^{2}+q\left(1+\varepsilon_{1} \varepsilon\right), \quad B=q\left(s^{2}-\Omega^{2}\right) .
$$

Equation (43) can be written as the modified Bessel equation form of order zero as

$$
\left(\frac{\mathrm{d}^{2}}{\mathrm{~d} r^{2}}+\frac{1}{r} \frac{\mathrm{d}}{\mathrm{d} r}-m_{1}^{2}\right)\left(\frac{\mathrm{d}^{2}}{\mathrm{~d} r^{2}}+\frac{1}{r} \frac{\mathrm{d}}{\mathrm{d} r}-m_{2}^{2}\right) \bar{\phi}=0 .
$$

The general solution of Eq. (46), which is bounded as $r \rightarrow \infty$, is given by

$$
\bar{\phi}=\sum_{i=1}^{2} A_{i} K_{0}\left(m_{i} r\right)
$$

where $A_{1}$ and $A_{2}$ represent arbitrary integration parameters determined from the boundary conditions and $K_{0}(\cdot)$ are modified Bessel's functions of order zero of the first kind. Accordingly, the solution of the function $\bar{\psi}$ is derived as

$$
\bar{\psi}=\frac{1}{\varepsilon_{1}} \sum_{i=1}^{2}\left(m_{i}^{2}-s^{2}\right) A_{i} K_{0}\left(m_{i} r\right) .
$$

Furthermore, using the Bessel function relation

$$
z K_{n}^{\prime}(z)=-z K_{n \pm 1}(z) \pm n K_{n}(z)
$$


leads to

$$
\bar{u}=-\sum_{i=1}^{2} m_{i} A_{i} K_{1}\left(m_{i} r\right) .
$$

After some mathematical manipulations, the thermal stress can be obtained as

$$
\left\{\bar{\sigma}_{r r}, \bar{\sigma}_{\xi \xi}, \bar{\sigma}_{z z}\right\}=\frac{1}{2 r} \sum_{i=1}^{2}\left\{\Psi_{i}, \Gamma_{i}, \Phi_{i}\right\} A_{i}
$$

where

$$
\begin{gathered}
\Psi_{i}=r\left(2 s^{2}-m_{i}^{2}\right) K_{0}\left(m_{i} r\right)-2 c_{1} m_{i} K_{1}\left(m_{i} r\right)+m_{i}^{2} r K_{2}\left(m_{i} r\right) \\
\Gamma_{i}=r\left[m_{i}^{2}\left(2+c_{1}\right)-2 s^{2}\right] K_{0}\left(m_{i} r\right)-2 m_{i} K_{1}\left(m_{i} r\right)+c_{1} m_{i}^{2} r K_{2}\left(m_{i} r\right) \\
\Phi_{i}=r\left[m_{i}^{2}\left(c_{3}-\frac{2 \varepsilon_{6}}{\varepsilon_{3}}\right)+\frac{2 \varepsilon_{6}}{\varepsilon_{3}} s^{2}\right] K_{0}\left(m_{i} r\right)-2 c_{4} m_{i} K_{1}\left(m_{i} r\right)+c_{3} m_{i}^{2} r K_{2}\left(m_{i} r\right) .
\end{gathered}
$$

From Eq. (17), we get the solution of temperature $\bar{\theta}$ as

$$
\bar{\theta}(r, s)=\frac{-1+\sqrt{1+2 k_{1} \bar{\psi}}}{k_{1}} .
$$

Also, Laplace's transform of the boundary conditions (29) and (31) gives

$$
\begin{gathered}
\bar{\psi}(R, s)=\theta_{0}\left(\frac{1}{s}+\frac{k_{1}}{2 s}\right)=\bar{G}(s), \\
\bar{\sigma}_{r r}(R, s)=0 .
\end{gathered}
$$

Using Eqs. (48) and (51) of the functions $\bar{\psi}$ and $\bar{\sigma}_{r r}$ into the boundary conditions (54) and (55), we obtain

$$
\begin{gathered}
\sum_{i=1}^{2}\left(m_{i}^{2}-s^{2}\right) A_{i} K_{0}\left(m_{i} R\right)=\varepsilon_{1} \bar{G}(s), \\
\sum_{i=1}^{2}\left[R\left(2 s^{2}-m_{i}^{2}\right) K_{0}\left(m_{i} R\right)-2 c_{1} m_{i} K_{1}\left(m_{i} R\right)+m_{i}^{2} R K_{2}\left(m_{i} R\right)\right] A_{i}=0 .
\end{gathered}
$$

Solution of the system of Eqs. (56) and (57) gives the values of the constants $A_{1}$ and $A_{2}$ completing the solution in the domain of Laplace's transform. 


\section{Numerical inversion of Laplace's transforms}

Keeping in mind the end goal to get the solutions in the physical domain, we convert Laplace's transform into the governing functions. We adopt a numerical reversal strategy that depends on a Fourier's series expansion [33]. In this technique, any function $\bar{g}(s)$ in Laplace domain can be modified to the time area $g(t)$ as

$$
g(t)=\frac{\mathrm{e}^{c t}}{t}\left\{\frac{1}{2} \bar{g}(c)+\operatorname{Re}\left[\sum_{n=1}^{N}(-1)^{n} \bar{g}\left(c+\frac{\mathrm{i} n \pi}{t}\right)\right]\right\}, \quad \mathrm{i}=\sqrt{-1} .
$$

For speedier convergence, various numerical trials have demonstrated that the estimation of $c$ fulfills the relation $c t \approx 4.7$ [34].

\section{Discussions of numerical results}

In this section, to explain the general solution behavior of the obtained theoretic results, we display some discussions and numerical results. For the purpose of numerical evaluations, we take cobalt as an orthotropic material. Values of the appropriate parameters of the material are [35]

$$
\begin{aligned}
& c_{11}=c_{22}=3.071 \cdot 10^{11} \mathrm{Nm}^{-1}, c_{12}=c_{13}=1.650 \cdot 10^{11} \mathrm{Nm}^{-1}, c_{23}=\frac{1}{2} c_{12}, \\
& \rho=8836 \mathrm{~kg} / \mathrm{m}^{3}, \beta_{11}=\beta_{22}=7.04 \cdot 10^{6} \mathrm{~N} / \mathrm{m}^{2} \mathrm{~K}, \beta_{33}=6.90 \cdot 10^{6} \mathrm{~N} / \mathrm{m}^{2} \mathrm{~K}, \\
& C_{E}=427 \mathrm{~J} / \mathrm{kg} \mathrm{K}, K_{r}=69 \mathrm{~W} / \mathrm{m} \mathrm{K}, T_{0}=298 \mathrm{~K} .
\end{aligned}
$$

Using the above material parameters, the distributions of dimensionless physical quantities; displacement $u$, temperature $\theta$ and thermal stressess $\sigma_{r r}$ and $\sigma_{\xi \xi}$ have been calculated mathematically and presented graphically in Figs. 1-12. Comparisons of numerical calculations were carried out for three cases.

\subsection{Effect of phase-lags}

Let us discuss how the non-dimensional temperature, displacement and thermal stresses vary with the phase-lag of the heat flux $\tau_{q}$ and the phase-lag of temperature gradient $\tau_{\theta}$ when the variability thermal conductivity parameter $k_{1}$ remains constant. In this case (Figs. 1-4), we take different values of the parameters $\tau_{q}$ and $\tau_{\theta}$. To get the governing equations of CTE theory from the introduced new model, we take $\left(\tau_{\theta}=\tau_{q}=0\right)$. To obtain the basic equations of LS model, we take $\left(\tau_{\theta}=0, \tau_{q}=0.1\right)$. Finally, in the case of generalized theory of thermoelasticity suggested by Tzou (DPL), we put $\left(\tau_{q}>0, \tau_{\theta}>0\right)$. We can conclude the following points from the demonstrated figures:

- The heat wave front moves forward with a finite speed in the medium with the passage of time. 


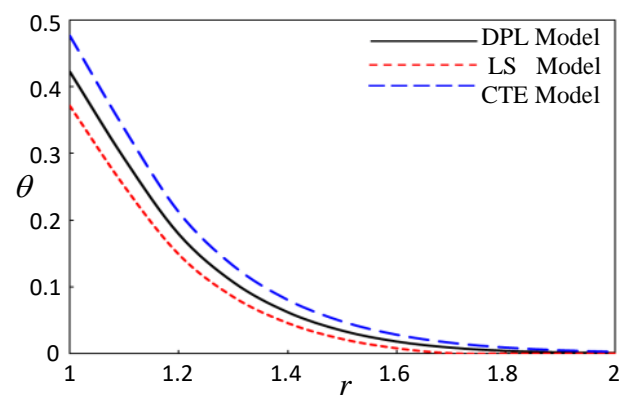

Fig. 1. Temperature distribution $\theta$ for different values of phase-lags $\tau_{q}$ and $\tau_{\theta}$

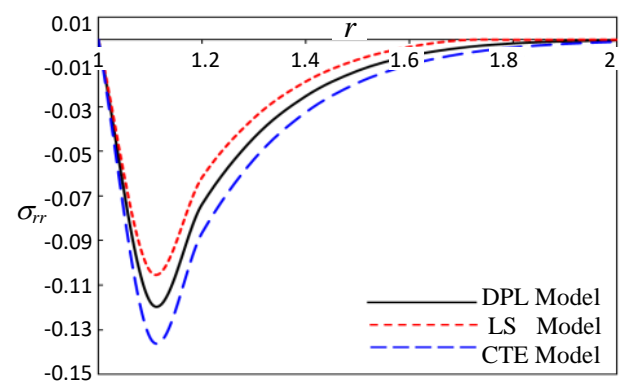

Fig. 3. Thermal stress distribution $\sigma_{r r}$ for different values of phase-lags $\tau_{q}$ and $\tau_{\theta}$

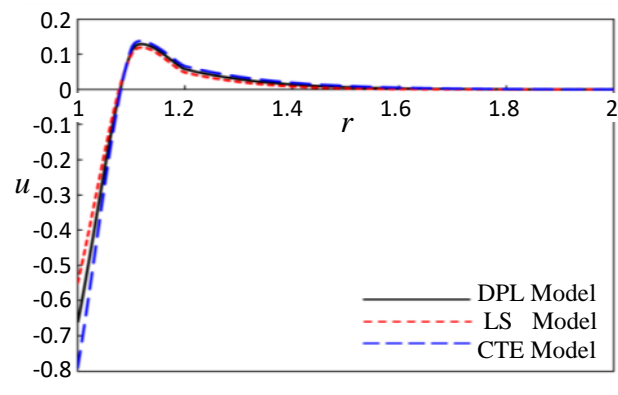

Fig. 2. Displacement distribution $u$ for different values of phase-lags $\tau_{q}$ and $\tau_{\theta}$

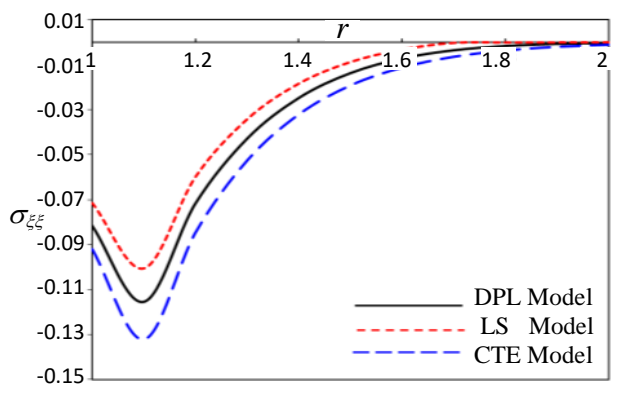

Fig. 4. Thermal stress distribution $\sigma_{\xi \xi}$ for different values of phase-lags $\tau_{q}$ and $\tau_{\theta}$

- The temperature distribution decreases as the space variable $r$ increases (Fig. 1).

- From Fig. 2, it is seen that value of displacement $u$ increases as $r$ decreases in the interval $1.1 \geqslant r \geqslant 0$ and decreases in the interval $1.6 \geqslant r \geqslant 1.1$, then it reaches to steady state when $r \geqslant 1.6$.

- From Figs. 3 and 4, it can be found that thermal stresses $\sigma_{r r}$ and $\sigma_{\xi \xi}$ increase as the distance $r$ increases. Also, it is clear that the most extreme estimations happen close to the surface of the hole and it decreases when $r$ increases.

- Near the surface of the cylinder, where the boundary conditions dominate, the coupled and the generalized theories give very close results. Inside the cylinder, the solution is markedly different. This is due to the fact that thermal waves in the coupled theory travel with an infinite speed of propagation as opposed to a finite speed in the generalized case.

- In all Figs. 1-4, it is observed that the phase-lag of the heat flux $\tau_{q}$ and the phase-lag of temperature gradient $\tau_{\theta}$ have significant effects on all fields.

- It is observed that all the waves reach the steady state depending on the values of the phase-lags $\tau_{q}$ and $\tau_{\theta}$.

- The variations of temperature for DPL theory is small in comparison to CTE theory. 
- The difference between the three curves at any fixed point as well as at fixed parameter $k_{1}$ for the three theories is clearly visible from these figures.

- The values in classical theory of thermoelasticity (CTE model) are different compared to those of other theories. The fact that in generalized thermoelasticity theories (DPL and LS), the waves propagate with finite speeds is evident in all figures. This validates clearly the difference between the modified theories of thermoelasticity and the classical coupled model.

- The behavior of three theories is generally quite similar. With the increase in distance, the results are quite close to each other, which is in agreement with the generalized theories of thermoelasticity.

\subsection{Effect of thermal conductivity parameter}

Investigating the variability thermal conductivity parameter $k_{1}$ effect on the non-dimensional temperature, displacement and thermal stresses when phase-lag of the heat flux $\tau_{q}$ and the phase-lag of temperature gradient $\tau_{\theta}$ remain constants. Here, we consider three distinct quantities of the parameter of variability thermal conductivity $k_{1}$ to examine the effect of temperature on thermal conductivity. We take $k_{1}=-1,-0.5$ when the thermal conductivity is dependent of temperature and $k_{1}=0$ for fixed thermal conductivity.

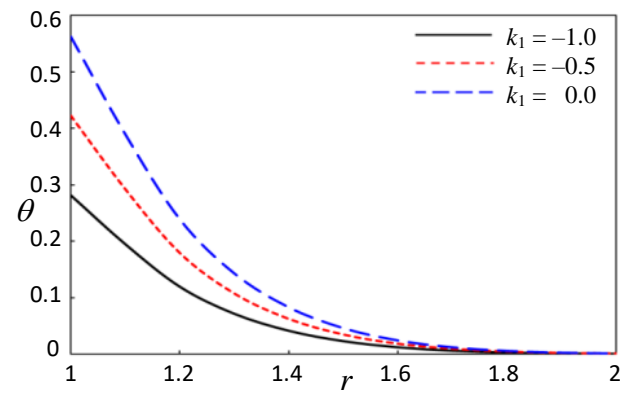

Fig. 5. Temperature distribution $\theta$ for different values of thermal conductivity parameter $k_{1}$

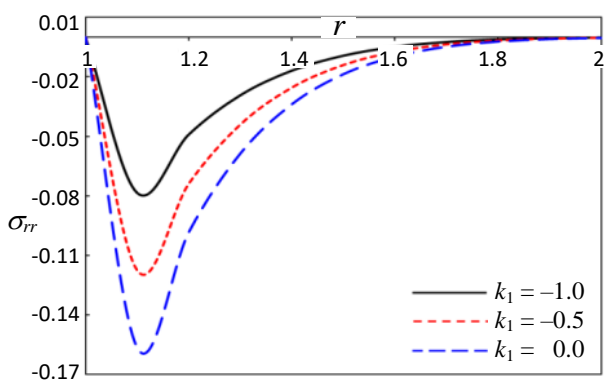

Fig. 7. Thermal stress distribution $\sigma_{r r}$ for different values of thermal conductivity parameter $k_{1}$

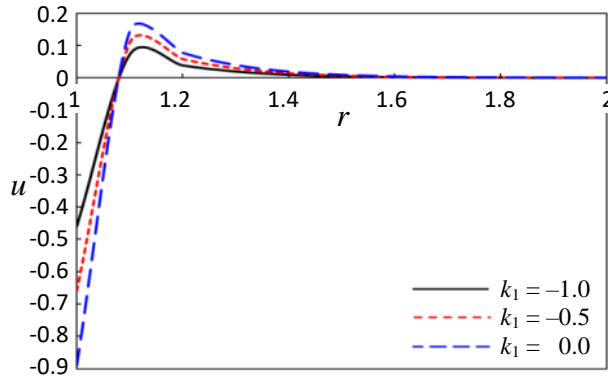

Fig. 6. Displacement distribution $u$ for different values of thermal conductivity parameter $k_{1}$

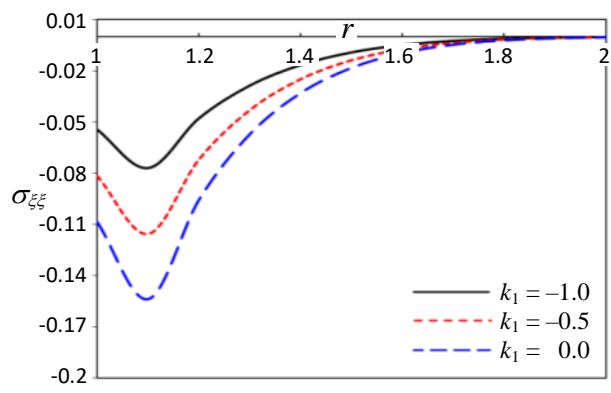

Fig. 8. Thermal stress distribution $\sigma_{\xi \xi}$ for different values of thermal conductivity parameter $k_{1}$ 
We selected the rotation parameter as $\Omega=5$ and lags $\tau_{q}=0.02$ and $\tau_{\theta}=0.01$ in this case. We also observe the following important facts from Figs. 5-8:

- The variability parameter $k_{1}$ has pronounced effects on all the studied fields.

- From Fig. 5, it is easily seen that the value of temperature increases with the increase of $k_{1}$ in all contexts of all theories of thermoelasticity and satisfied the considered boundary conditions.

- From Fig. 6, it is shown that the amplitude of distribution of the displacement $u$ rises with the increase of $k_{1}$.

- From Figs. 7 and 8, it can be found that the absolute values of thermal stresses $\sigma_{r r}$ and $\sigma_{\xi \xi}$ increase as the parameter $k_{1}$ decreases.

- We have noticed from these figures that the variability thermal conductivity parameter $k_{1}$ has a significant effect on all the fields which add an importance to our consideration about the thermal conductivity to be variable.

- From these figures, we find that the field quantities depend not only on the state and space variables $t$ and $r$, but also on the variability thermal conductivity parameter and phase-lags parameters. The phenomenon of finite speeds of propagation is manifested in all figures.

\subsection{Effect of rotation}

Studying the effect of rotation on dimensionless physical quantities when phase-lag of the heat flux $\tau_{q}$ and the phase-lag of temperature gradient $\tau_{\theta}$ and the variability thermal conductivity parameter $k_{1}$ remain constants. In this case, Figs. 9-12 show the variations of temperature, displacement and thermal stresses along the radial direction at various values of rotation parameter $\Omega$. From these figures it is observed that:

- The amplitude of the temperature has a slight increase for the rotating case in comparison with the non-rotating case due to the presence of rotation term (see Fig. 9).

- It is clear from the graph in Fig. 10 that the variations of displacement with the varied values of the rotation parameter $\Omega$ in the context of phase-lag model is close in both rotating and non-rotating case.

- Also, a significant difference in thermal stresses is noticed for different values of rotation parameter $\Omega$ (see Figs. 11 and 12).

- Consequently, the idea of rotation in a thermoelastic orthotropic medium with the parameters of phase-lags will yield more destruction as contrast with non-rotating type.

- Therefore, the presence of rotation in current model is of significance.

- Rotation will play its role in the wave propagations that has been appeared by looking at the distributions of various waves in a rotating and non-rotating thermoelastic orthotropic medium.

- As detected from the introduced graphical results, the parameter of rotation plays a significant role on the deformation variants in the body. 


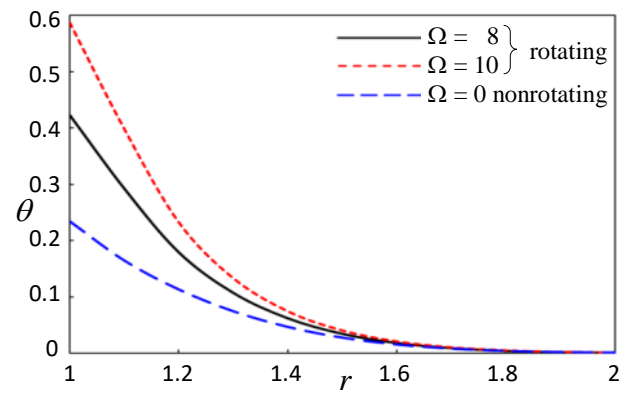

Fig. 9. Temperature distribution $\theta$ for different values of angular velocity (rotation) $\Omega$

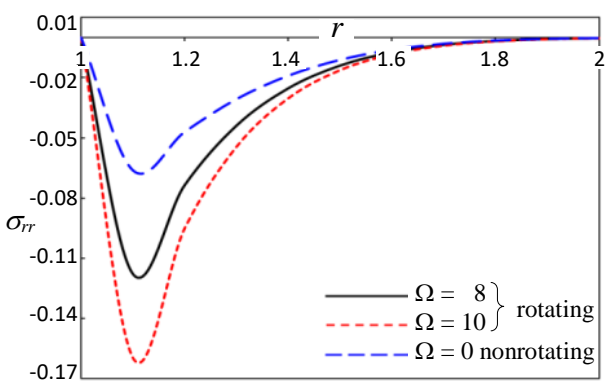

Fig. 11. Thermal stress distribution $\sigma_{r r}$ for different values of angular velocity (rotation) $\Omega$

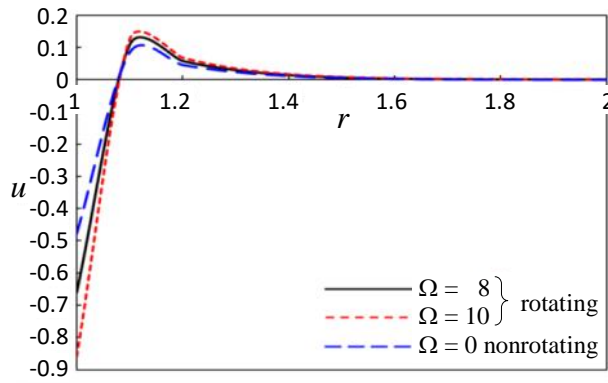

Fig. 10. Displacement distribution $u$ for different values ofangular velocity (rotation) $\Omega$

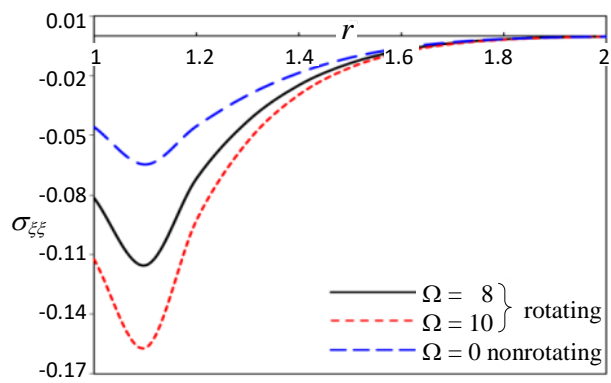

Fig. 12. Thermal stress distribution $\sigma \xi \xi$ for different values ofangular velocity (rotation) $\Omega$

\section{Conclusions}

In this work, we have investigated a one-dimensional problem for an infinite homogeneous orthotropic thermally conducting body containing a cylindrical cavity affected by the angular velocity under thermal shock surface heating using the method of Laplace's transform. From the obtained numerical results, some main conclusions are given by:

- The studied field quantities depend on time $t$ and space $r$ and on the variability of thermal conductivity, rotation and phase-lags parameters.

- The dependence of thermal conductivity on the temperature has significant effects on the velocity of propagation of waves and mechanical interactions.

- In the introduced model, the presence of rotation terms is essential.

- When the considered medium rotates with some angular velocity, the values of temperature distribution are much smaller in magnitude.

- The idea of rotation in a thermoelastic orthotropic medium will yield more pulverization as contrast with non-rotating sort.

- The theories of Biot and generalized thermoelasticity proposed by Lord and Shulman can be obtained as special cases from the current model. 
- The results displayed in this work should prove to be valuable for researchers in scientific and designing, in addition, for those working on the improvement of mechanics of materials.

\title{
Acknowledgements
}

This project was funded by the Deanship of Scientific Research (DSR) at King Abdulaziz University, Jeddah, under grant no. G-199-130-36. The authors, therefore, acknowledge with thanks DSR for technical and financial support.

\author{
Manuscript received by Editorial Board, March 11, 2017; \\ final version, July 26, 2017.
}

\section{References}

[1] R. Berman. The thermal conductivity of dielectric solids at low temperatures. Advances in Physics, 2(5):103-140, 1953. doi: 10.1080/00018735300101192.

[2] M.A. Biot. Thermoelasticity and irreversible thermodynamics. Journal of Applied Physics, 27(3):240-253, 1956. doi: 10.1063/1.1722351.

[3] H.W. Lord and Y. Shulman. A generalized dynamical theory of thermoelasticity. Journal of the Mechanics and Physics of Solids, 15(5):299-309, 1967. doi: 10.1016/0022-5096(67)90024-5.

[4] A.E. Green and K.A. Lindsay. Thermoelasticity. Journal of Elasticity, 2(1):1-7, 1972. doi: 10.1007/BF00045689.

[5] A.M. Zenkour. Three-dimensional thermal shock plate problem within the framework of different thermoelasticity theories. Composite Structures, 132:1029-1042, 2015. doi: 10.1016/j.compstruct.2015.07.013.

[6] A.M. Zenkour. Nonlocal thermoelasticity theory without energy dissipation for nano-machined beam resonators subjected to various boundary conditions. Microsystem Technologies, 23(1):55-65, 2017. doi: 10.1007/s00542-015-2703-4.

[7] A.M. Zenkour. Thermoelastic response of a microbeam embedded in visco-Pasternak's medium based on GN-III model. Journal of Thermal Stresses, 40(2):198-210, 2017. doi: 10.1080/01495739.2016.1249039.

[8] A.M. Zenkour. Vibration analysis of generalized thermoelastic microbeams resting on viscoPasternak's foundations. Advances in Aircraft and Spacecraft Science, 4(3):269-280, 2017. doi: 10.12989/aas.2017.4.3.269.

[9] D.Y. Tzou. A unified field approach for heat conduction from macro-to micro-scales. Journal of Heat Transfer, 117(1):8-16, 1995. doi: 10.1115/1.2822329.

[10] A.M. Zenkour. Two-dimensional coupled solution for thermoelastic beams via generalized dual-phase-lags model. Mathematical Modelling and Analysis, 21(3):319-335, 2016. doi: 10.3846/13926292.2016.1157835.

[11] A.E. Abouelregal. Fractional order generalized thermo-piezoelectric semi-infinite medium with temperature-dependent properties subjected to a ramp-type heating. Journal of Thermal Stresses, 34(11):1139-1155, 2011. doi: 10.1080/01495739.2011.606018.

[12] A.M. Zenkour and A.E. Abouelregal. Nonlocal thermoelastic vibrations for variable thermal conductivity nanobeams due to harmonically varying heat. Journal of Vibroengineering, 16(8):3665-3678, 2014. 
[13] A.M. Zenkour, A.E. Abouelregal, K.A. Alnefaie, X. Zhang, and E.C. Aifantis. Nonlocal thermoelasticity theory for thermal-shock nanobeams with temperature-dependent thermal conductivity. Journal of Thermal Stresses, 38(9):1049-1067, 2015. doi: 10.1080/01495739.2015.1038490.

[14] M.A. Ezzat and A.A. El-Bary. Effects of variable thermal conductivity and fractional order of heat transfer on a perfect conducting infinitely long hollow cylinder. International Journal of Thermal Sciences, 108:62-69, 2016. doi: 10.1016/j.ijthermalsci.2016.04.020.

[15] A.E. Abouelregal and S.M. Abo-Dahab. Dual phase lag model on magneto-thermoelasticity infinite non-homogeneous solid having a spherical cavity. Journal of Thermal Stresses, 35(9):820841, 2012. doi: 10.1080/01495739.2012.697838.

[16] A.E. Abouelregal and S.M. Abo-Dahab. Dual-phase-lag diffusion model for Thomson's phenomenon on electromagneto-thermoelastic an infinitely long solid cylinder. Journal of Computational and Theoretical Nanoscience, 11(4):1031-1039, 2014. doi: 10.1166/jctn.2014.3459.

[17] A.E. Abouelregal and A.M. Zenkour. The effect of fractional thermoelasticity on a twodimensional problem of a mode $i$ crack in a rotating fiber-reinforced thermoelastic medium. Chinese Physics B, 22(10):108102, 2013.

[18] R. Singh and V. Kumar. Eigen value approach to two dimensional problem in generalized magneto micropolar thermoelastic medium with rotation effect. International Journal of Applied Mechanics and Engineering, 21(1):205-219, 2016. doi: 10.1515/ijame-2016-0013.

[19] C. Xiong and Y. Guo. Effect of variable properties and moving heat source on magnetothermoelastic problem under fractional order thermoelasticity. Advances in Materials Science and Engineering, 2016:5341569, 2016. doi: 10.1155/2016/5341569.

[20] R. Kumar, K. Singh, and D. Pathania. Interactions due to hall current and rotation in a magnetomicropolar fractional order thermoelastic half-space subjected to ramp-type heating. Multidiscipline Modeling in Materials and Structures, 12(1):133-150, 2016. doi: 10.1108/MMMS-032015-0016.

[21] H.H. Sherief and F.A. Hamza. Modeling of variable thermal conductivity in a generalized thermoelastic infinitely long hollow cylinder. Meccanica, 51(3):551-558, 2016. doi: 10.1007/s11012-015-0219-8.

[22] A.M. Zenkour and A.E. Abouelregal. Effects of phase-lags in a thermoviscoelastic orthotropic continuum with a cylindrical hole and variable thermal conductivity. Archives of Mechanics, 67(6):457-475, 2015.

[23] A.M. Zenkour and A.E. Abouelregal. Non-simple magnetothermoelastic solid cylinder with variable thermal conductivity due to harmonically varying heat. Earthquakes and Structures, 10(3):681-697, 2016. doi: 10.12989/eas.2016.10.3.681.

[24] A.M. Zenkour. Effect of temperature-dependent physical properties on nanobeam structures induced by ramp-type heating. KSCE Journal of Civil Engineering, 21(5):1820-1828, 2017. doi: 10.1007/s12205-016-1004-5.

[25] J.N. Sharma and V. Walia. Effect of rotation on Rayleigh waves in piezothermoelastic half space. International Journal of Solids and Structures, 44(3):1060-1072, 2007. doi: 10.1016/j.ijsolstr.2006.06.005.

[26] S.G. Lekhnitskij. Theory of the Elasticity of Anisotropic Bodies. Mir Publishers, Mocow, 1981.

[27] B.A. Boley and J.H. Weiner. Theory of Thermal Stresses. John Wiley \& Sons, New York, 1960.

[28] F. Szuecs, M. Werner, R.S. Sussmann, C.S.J. Pickles, and H.J. Fecht. Temperature dependence of Young's modulus and degradation of chemical vapor deposited diamond. Journal of Applied Physics, 86(11):6010-6017, 1999. doi: 10.1063/1.371648.

[29] O.R. Budaev, M.N. Ivanova, and B.B. Damdinov. Temperature dependence of shear elasticity of some liquids. Advances in Colloid and Interface Science, 104(1):307-310, 2003. doi: 10.1016/S0001-8686(03)00050-2. 
[30] V.V. Rishin, B.A. Lyashenko, K.G. Akinin, and G.N. Nadezhdin. Temperature dependence of adhesion strength and elasticity of some heat-resistant coatings. Strength of Materials, 5(1):123-126, 1973. doi: 10.1007/BF00762888.

[31] S.S. Manson. Behavior of materials under conditions of thermal stress. NACA Technical Report 1170, 1954.

[32] N. Noda. Thermal stresses in materials with temperature-dependent properties. In R.B. Hetnarski, editor, Thermal Stresses I, chapter 6. North-Holland, Amsterdam, 1986.

[33] G. Honig and U. Hirdes. A method for the numerical inversion of Laplace transforms. Journal of Computational and Applied Mathematics, 10(1):113-132, 1984. doi: 10.1016/03770427(84)90075-X.

[34] D.Y. Tzou. Macro-To Microscale Heat Transfer: The Lagging Behavior. Taylor and Francis, Washington DC, 1996.

[35] J.C. Misra, N.C. Chattopadhyay, and S.C. Samanta. Study of the thermoelastic interactions in an elastic half space subjected to a ramp-type heating - A state-space approach. International Journal of Engineering Science, 34(5):579-596, 1996. doi: 10.1016/0020-7225(95)00128-X. 